\title{
Modelo de asociatividad en la cadena productiva en las Mipymes agroindustriales
}

\author{
Model of associativity in the production chain in Agroindustrial SMEs \\ Lila Margarita Bada Carbajal ${ }^{\mathrm{a}, *}$, Luis Arturo Rivas Tovar $^{\mathrm{b}}$ \\ y Herman Frank Littlewood Zimmerman ${ }^{\mathrm{c}}$ \\ a Instituto Tecnológico Superior de Álamo Temapache, México \\ ${ }^{\mathrm{b}}$ Instituto Politécnico Nacional, México \\ ${ }^{\mathrm{c}}$ Instituto Tecnológico de Estudios Superiores Monterrey, México
}

Recibido el 3 de julio de 2015; aceptado el 18 de enero de 2016

Disponible en Internet el 18 de agosto de 2017

\section{Resumen}

Esta investigación tiene como objetivo proponer un modelo de asociatividad en cadena productiva de las micro, pequeñas y medianas empresas (MIPYMES) agroindustriales de cítricos en el norte del estado de Veracruz, México, con el propósito de explicar en qué medida los actores directos, los servicios de apoyo, el entorno, las relaciones y las políticas de gobierno determinan la asociatividad en la cadena productiva. La problemática que origina esta investigación es el desconocimiento del funcionamiento de las MIPYMES agroindustriales de cítricos en esta zona del país. El resultado de esta investigación es un modelo que representa el funcionamiento de estas empresas en asociatividad con la cadena productiva considerando los elementos que la forman, proponiendo alternativas para generar una mayor cooperación o coalición de las empresas que interactúan para obtener beneficios mutuos.

(C) 2017 Universidad Nacional Autónoma de México, Facultad de Contaduría y Administración. Este es un artículo Open Access bajo la licencia CC BY-NC-ND (http://creativecommons.org/licenses/by-nc-nd/4.0/).

Palabras clave: Asociatividad; Cadena productiva; Cítricos

Códigos JEL: L22; L23; M11

\footnotetext{
* Autor para correspondencia.

Correo electrónico: limbac@ @otmail.com (L.M. Bada Carbajal).

La revisión por pares es responsabilidad de la Universidad Nacional Autónoma de México.
} 


\begin{abstract}
This research aims to propose an associativity model in the production chain of citrus agro industrial micro, small and medium enterprises in the northern part of the state of Veracruz, Mexico. The goal is to explain to what degree direct actors, support services, environment, government relations and policies determine associativity in the production chain. The problem frame which gives rise to this study is the lack of knowledge about citrus agro industrial micro and SME's in this region of the country. The outcome of the research is a model which represents functioning of these businesses in associativity with the production chain considering elements which make it up, and the proposal of alternatives to generate a greater cooperation or coalition of interacting industries in order to obtain mutual benefits.

(C) 2017 Universidad Nacional Autónoma de México, Facultad de Contaduría y Administración. This is an open access article under the CC BY-NC-ND license (http://creativecommons.org/licenses/by-nc-nd/4.0/).
\end{abstract}

Keywords: Associativity; Production chain; Citrus

JEL classification: L22; L23; M11

\title{
Introducción
}

Esta investigación tiene como objetivo proponer un modelo de asociatividad en cadena productiva de las micro, pequeñas y medianas empresas (MIPYMES) agroindustriales en cítricos del norte del estado de Veracruz, debido a que no existe un modelo que represente la cadena productiva en este contexto. Los criterios bajo los cuales se construyó fueron los siguientes: primeramente se elaboró un modelo ex ante basado en el la evidencia empírica del estado del arte, posteriormente se establece el modelo ex post facto donde se establecen la relaciones significativas entre las variables a través de correlaciones de Pearson y $\mathrm{R}^{2}$, así como el análisis de factores utilizando el método de extracción y el modelamiento de ecuaciones estructurales para determinar las variables del modelo. Con la determinación de las variables que van a estar en el modelo y matrices de información que se utilizaron para recolectar información cualitativa, se realiza el mapeo del modelo de asociatividad en la cadena productiva de las MIPYMES de cítricos en Veracruz. Los apartados de los que se compone la investigación son los siguientes: en el primer apartado se expone el contexto de las MIPYMES agroindustriales en cítricos del norte del estado de Veracruz. En el segundo apartado se desarrollan los aspectos teóricos en los que se fundamenta la investigación sobre cadenas productivas y los modelos de asociatividad. El tercer apartado desarrolla la metodología. En el cuarto apartado se lleva a cabo el análisis y los resultados obtenidos a través del instrumento de medición realizando los análisis estadísticos para obtener las variables del modelo, se elabora la propuesta del modelo de asociatividad en cadena productiva de las MIPYMES agroindustriales en cítricos del norte del estado de Veracruz; en este apartado se detallan los resultados cuantitativos y cualitativos, con base en los análisis llevados a cabo, que a su vez permitieron alcanzar el objetivo principal de la investigación. Por último, en el quinto apartado se exponen las conclusiones y recomendaciones.

\section{Micro, pequeñas y medianas empresas agroindustriales en el estado de Veracruz}

México es el quinto productor mundial de cítricos (4.6\% del total), detrás de China (21\%), Brasil (18\%), Estados Unidos (11\%) y la India (6\%) (Secretaría de Agricultura, 2014). 
Tabla 1

Municipios más representativos en MIPYMES en Veracruz

\begin{tabular}{lc}
\hline Municipios de estado de Veracruz & MIPYMES \\
\hline Córdoba & 3,781 \\
Xalapa & 3,114 \\
Veracruz & 2,609 \\
Tuxpan & 1,356 \\
Poza Rica & 1,213 \\
Orizaba & 1,212 \\
Coatzacoalcos & 1,193 \\
Boca el Río & 607 \\
Fortín & 589 \\
Minatitlán & 561 \\
\hline
\end{tabular}

Fuente: elaboración propia con base al Sistema de Información Empresarial Mexicano (SIEM, 2014).

La actividad citrícola reviste gran importancia para Veracruz, pues es el principal productor de cítricos en México.

El estado de Veracruz, ubicado del noroeste al sureste sobre la costa en México, cuenta con 212 municipios y está constituido por 10 regiones: Huasteca Alta, Huasteca Baja, Totonaca, Nautla, Capital, Sotavento, Montañas, Papaloapan, de los Tuxtlas y Olmeca.

De acuerdo al Sistema de Información Empresarial Mexicano (SIEM, 2014), los municipios más representativos en cuanto a las MIPYMES registradas (tabla 1) son Córdoba, Xalapa y el puerto de Veracruz, con actividades de comercio y portuarias, situadas en el centro del estado; Tuxpan y Poza Rica, ubicadas en la zona norte del estado, con actividades de comercio y petróleo; los municipios de Orizaba, Coatzacoalcos, Boca del Río, Fortín y Minatitlán tienen actividades industriales y comerciales principalmente y actividad portuaria.

En la zona norte del estado de Veracruz se realizan actividades económicas, comerciales, industriales y de servicios, principalmente del sector agropecuario (agricultura, ganadería, silvicultura y pesca), que tienen una fuerte influencia de MIPYMES agroindustriales que han permitido a la región realizar gran variedad de productos procesados ofreciendo un valor agregado a los productos del sector agropecuario.

La agroindustria es una actividad muy compleja; en ella interactúa lo agrícola con lo industrial, por lo que requiere desarrollos tecnológicos y requerimientos de capital, sistemas de distribución y comercialización cada vez más sofisticados y necesarios para llegar al consumidor, que es quien acepta o rechaza los productos y define los grados de transformación (Romero, 2001).

La agroindustria en cítricos está comprendida por empresas dedicadas al cepillado, empacado y encerado de cítricos, extracción de jugo, concentración de jugo, extracción de aceite, extracción de pectina y deshidratación de cáscara; dichas empresas o establecimientos no están claramente identificados en la información censal disponible, ya que son fuentes de ingresos económicos y la mayoría de las empresas —en el caso de las micro y pequeñas - se encuentran registradas fiscalmente como personas físicas con actividades empresariales o régimen de incorporación fiscal teniendo poco tiempo de creación, donde la mayoría de ellas, dependiendo de su situación económica, se dan de baja temporal o definitiva ante la Secretaria de Administración Tributaria (SAT), lo que dificulta llevar un censo específico.

En el estado de Veracruz, la producción de cítricos (principalmente naranja, limón, tangerina, toronja y mandarina) obtuvo conjuntamente en 2014 una producción de 3,560,580.47 toneladas. 
Los municipios productores de naranja se encuentran principalmente en la zona norte del estado de Veracruz. El municipio de Álamo Temapache es el principal productor de naranja, mandarina y tangerina, con una producción de 2,353,699.60, 154,595.20 y 178,900.21 toneladas, respectivamente, y la ciudad de Martínez de la Torre es el principal productor de toronja y limón, con una producción de 250,353.40 y 623,062.06 toneladas, respectivamente (Sistema de Información Agropecuaria y Pesquera (2014).

El contexto donde se desarrolló esta investigación fueron las MIPYMES agroindustriales del norte del estado de Veracruz de los municipios de Álamo Temapache, Papantla, Gutiérrez Zamora y Poza Rica, ubicadas en las regiones Huasteca Baja y Totonaca, puesto que son los principales municipios productores de cítricos y con mayor número de agroindustrias registradas en el estado.

\section{Asociatividad en la cadena productiva}

Una estrategia clave para el desarrollo de las MIPYMES en el mundo globalizado es fomentar la asociatividad de las empresas, promoviendo la creación de clusters y redes empresariales en cadenas productivas competitivas. El estudio de la asociatividad y las cadenas productivas ha sido abordado por numerosos estudios, entre los que destacan los siguientes:

López y Calderón (2006, p.14) definen la asociatividad como «una estrategia resultado de una cooperación o coalición de empresas en función de un objetivo en común, en la que cada participante mantiene independencia jurídica y gerencial».

De acuerdo con Dini (2003), las formas de asociatividad son: cadenas productivas, redes y clusters.

\section{Cadena productiva}

Las cadenas productivas surgen como una alternativa de eficiencia colectiva, pero su desarrollo requiere políticas macroeconómicas coherentes, identificación de las ventajas competitivas y un entorno que genere estabilidad y confianza.

A continuación se desarrolla el concepto de cadena productiva desde la perspectiva de varios autores e instituciones.

El Programa Estatal de Ciencia y Tecnología del Estado de Jalisco (2007, p. 87), México, define la cadena productiva como «el proceso que sigue un producto o servicio a través de las actividades de producción, transformación e intercambio, hasta llegar al consumidor final. Incluye además el abasto de insumos (financiamiento, seguros, maquinaria, equipo, materias primas directas e indirectas, etc.) y sistemas relevantes, así como todos los servicios que afectan de manera significativa a dichas actividades: investigación y desarrollo y asistencia técnica, entre otros, para realizar actividades competitivas y sostenibles que permita generar riqueza material para incrementar el nivel de bienestar».

Wisner (2003) y Croxton, García-Dastugue, Lambert y Rogers (2001, p. 24) conceptualizan la cadena productiva como «la integración de los procesos clave de negocios que ocurren dentro de la red conformada por los proveedores de insumos, los fabricantes, los distribuidores y los minoristas independientes, cuyo objetivo es optimizar el flujo de los bienes, servicios e información».

De acuerdo con la Comisión Económica de América latina y el Caribe (CEPAL), (2003, p. 112), el concepto de cadenas productivas «implica la concentración sectorial y/o geográfica de empresas que desempeñan las mismas actividades estrechamente relacionadas entre sí (tanto 
hacia atrás como hacia delante) con importantes y acumulativas economías externas y posibilidad de llevar a cabo una acción conjunta en la búsqueda de la eficiencia colectiva».

Desde el punto de vista legal, el Diario Oficial de la Federación en la Ley para el desarrollo de la competitividad de la MIPYME (2009, p. 19) define a las cadenas productivas como «sistemas productivos que integran conjuntos de empresas que añaden valor agregado a productos y servicios a través de las fases del proceso económico».

Lazzarini, Chaddad y Cook (2001, p. 142) conceptualizan la cadena productiva como «el conjunto secuencial de actores que participan en las transacciones sucesivas para la generación de un bien o servicio, incluyendo el sector primario hasta el consumidor final y los servicios proveídos a lo largo de la cadena».

Kaplinsky (2000, p. 76) define la cadena productiva como «una herramienta de análisis que permite identificar los principales puntos críticos y potenciales de desarrollo, para luego definir e impulsar estrategias concentradas en los actores involucrados».

Con base en lo anterior, podemos definir el concepto de cadena productiva como «un sistema productivo que integra un conjunto de actores y relaciones secuenciales de negocios, servicios relevantes y demás elementos que intervienen en el proceso de elaboración de un producto desde el sector primario hasta el consumidor final y los servicios proveídos a lo largo de la cadena, para luego definir estrategias en los actores involucrados».

\section{Modelos de cadenas productivas}

Los modelos de cadenas productivas reportados en el estado del arte son los globales y los sectoriales.

\section{Modelos de cadenas productivas globales}

De acuerdo con Gereffi (1999), las cadenas productivas globales darían a entender que la cuasijerarquía donde los fabricantes y compradores desempeñan el papel principal domina el grupo de las manufacturas tradicionales. En algunos casos coexisten cadenas productivas diferentes, con empresas que participan tanto en una cadena productiva local como en una global. Existen dos tipos de cadenas productivas globales:

1. Cadenas productivas destinadas al productor.

2. Cadenas productivas destinadas al comprador.

\section{Modelos de cadenas productivas sectoriales}

Estos modelos de cadenas productivas sectoriales están integrados por los tres sectores económicos de la economía mexicana: el sector agropecuario, el industrial y el de servicios. Estos sectores tienen relaciones intersectoriales debido a que el sector agropecuario vende materias primas al sector industrial y le compra fertilizantes, abonos y maquinaria. El sector servicios compra alimentos al sector agropecuario, y este solicita servicios financieros, comerciales y de transporte al sector servicios. El sector industrial vende al sector servicios muebles, equipo de oficina, camiones, etc., y el sector servicios proporciona a la industria servicios profesionales, médicos, financieros, etc.

Los modelos de cadenas productivas agroindustriales tienen relaciones intersectoriales con las actividades agropecuarias e industriales y demuestran la importancia de la tecnología en el acercamiento del productor al consumidor final, vía tecnología desde el punto de vista de venta, 
apoyadas fuertemente en tecnologías de información (Programa Estatal de Ciencia y Tecnología del Estado de Jalisco, 2007).

Los estudios sobre cadenas productivas tienen sus orígenes en Europa en los años setenta y permitieron mejorar la competitividad de varios productos como leche, carne y vino, promoviendo la definición de políticas sectoriales consensuadas entre los diferentes actores de la cadena (Van der Heyden y Camacho, 2006).

Algunos países han desarrollado proyectos de integración productiva, a través de sus gobiernos, para construir políticas de desarrollo articuladas, y a través de sus universidades, para elaborar investigaciones de integración regional.

En Estados Unidos existen trabajos sobre cadenas productivas elaborados por el Banco Interamericano de Desarrollo de Washington D.C. (Pietrobelli y Rabellotti, 2005; Guaipatín, 2004) donde alinean los factores clave que promueven la innovación en: educación, acceso al crédito, existencia de instituciones efectivas y apertura económica.

Así mismo, la Universidad de Duke, Durham, Carolina del Norte de Estados Unidos (Gereffi, 1999), y la Universidad de Ohio y Nevada en Reno (Croxton et al., 2001) han realizado investigaciones sobre cadenas productivas para estudiar las múltiples dimensiones de ascenso industrial, el cual constituye una nueva forma de análisis del desarrollo económico en la era de una industrialización orientada a las exportaciones, donde se establecen implicaciones teóricas para el desarrollo del enfoque de las cadenas productivas con bases históricas y organizativas.

En los países latinoamericanos el enfoque de cadenas productivas es relativamente nuevo, y a partir del año 2000 se han realizado estudios de cadenas productivas en países como Colombia, Perú, Bolivia, Costa Rica, Brasil, Argentina, Venezuela y México, donde a través de sus instituciones gubernamentales y educativas de posgrado e investigación han demostrado que el enfoque de cadena productiva es pertinente en el contexto actual de evolución de la economía mundial, competitividad, productividad, globalización, innovación tecnológica y complejos sistemas agroalimentarios, por lo cual el enfoque permite dar una mirada sistemática a las actividades productivas.

En México se han realizado estudios de cadenas productivas a partir del año 2002, básicamente a través de instituciones gubernamentales como la Secretaría de Economía, la Secretaría de Agricultura, Ganadería, Apicultura y Pesca, la Secretaría de la Pequeña y Mediana Empresa, la Nacional Financiera, la Institución Nacional de Investigaciones Forestales, Agrícolas y Pecuarias, el Programa Estatal de la Ciencia y Tecnología de Jalisco y el Consejo Nacional para la Competitividad de la Micro, Pequeña y Mediana Empresa, que han permitido el establecimiento de planes sectoriales y regionales, programas de competitividad para incrementar la productividad y la competitividad de las MIPYMES a través de la integración en cadenas productivas.

Las instituciones educativas de nivel superior y posgrado en México han realizado estudios sobre cadenas productivas. El Instituto Tecnológico de Estudios Superiores de Monterrey (2004), la Universidad de Aguascalientes (Carranza et al., 2007) y la Universidad Autónoma de Chapingo (Cuevas et al., 2007) han realizado investigaciones que tienen como objetivo promover la movilización de recursos productivos existentes en las zonas rurales, a través de una mejor vinculación de los pequeños productores en las cadenas productivas, con el fin de analizar el desempeño de la cadena productiva e identificar sus factores críticos y potenciales de desarrollo.

En el ámbito académico solo existen trabajos de reflexión teórica, como el de Ochoa y Montoya, (2010), quienes realizan una metáfora biológica aplicada a la asociatividad empresarial en cadenas productivas agropecuarias. Recopila diversas aplicaciones de la metáfora como metodología para el estudio de las organizaciones y sus problemas, así como la naturaleza, las características y los fundamentos tanto de los consorcios microbianos como de los encadenamientos productivos 
agropecuarios. Este trabajo concluye exponiendo las ventajas y limitaciones de esta metodología conceptual para el caso de las cadenas productivas agropecuarias sin ofrecer ninguna evidencia empírica. La relevancia de este trabajo que presentamos es ofrecer evidencia concreta de las MIPYMES agroindustriales en cítricos más representativas en Veracruz, México.

El objetivo de la investigación busca responder esta pregunta: ¿Qué modelo explica la asociatividad de la cadena productiva de las MIPYMES agroindustriales de cítricos ubicadas en la parte norte del estado de Veracruz?

En la figura 1 se muestra el modelo ex ante que se construye con base en la evidencia empírica reportada en el estado del arte. Cada variable independiente - actores directos, entorno, servicios de apoyo, relaciones y políticas de gobierno - tiene su fundamentación teórica, y de la misma manera la variable dependiente: asociatividad. La operacionalización de las variables se realizó tomando en cuenta la variable, la definición conceptual, las dimensiones y los indicadores.

\section{Método de investigación}

La figura 2 muestra el método hipotético deductivo, donde se aprecia la secuencia de pasos para llevar a cabo la investigación.

En la figura 3 se observa la congruencia metodológica del título, con el planteamiento del problema, objetivos generales y específicos, así como las preguntas de investigación, hipótesis general e hipótesis de trabajo, cada uno de estos elementos alineados a cada variable independiente.

Los sujetos de investigación a quienes se les aplicó el instrumento de medición son los gerentes y dueños de las MIPYMES agroindustriales en cítricos del norte del estado de Veracruz ${ }^{1}$, de las ciudades de Álamo Temapache, Papantla, Gutiérrez Zamora y Poza Rica, en las agroindustrias de cepillado, encerado y empacado, extracción de jugo, concentrado de jugo, extracción de aceite, deshidratación de cáscara de naranja y extracción de pectina. El tipo de muestreo utilizado es probabilístico, porque se conoce la población, que son 53 MIPYMES $^{2}$.

En este sentido, la muestra se determina con base en la tabla de Krejcie y Morgan (1970), donde el tamaño de la población y la cantidad de errores determinan el tamaño de la muestra seleccionada al azar. Estos autores establecen una fórmula para estimar el tamaño de la muestra y con base en ella construyen la tabla donde se determina una muestra en relación a una población.

Fórmula:

$$
s=\frac{X^{2} N P(1-P)}{\left.d^{2}(N-1) 1\right)+X^{2} P(1-P)}
$$

Ubicada la población en la tabla, tenemos que con una población de 55 (que es el dato que más se acerca nuestra población en la tabla) se tiene una muestra de 48 empresas.

Se diseñó un instrumento de medición tomando como base lo siguiente: diagrama de variables, diagrama sagital de variables, matriz metodológica de variables (definición conceptual, definición operacional, dimensiones, indicadores y los ítems), matriz de validez de contenido y nivel de medi-

\footnotetext{
${ }^{1}$ Comprendidas en las regiones Huasteca Baja y Totonaca, que son la zona donde se encuentra la mayor producción de cítricos y de agroindustrias procesadoras.

${ }^{2}$ Integrada por 22 microempresas de jugo simple, 24 micro y pequeñas empresas cepilladoras, enceradoras y empacadoras de cítricos, y 7 pequeñas y medianas empresas procesadoras de jugo concentrado, aceite esencial, cáscara deshidratada y pectina.
} 


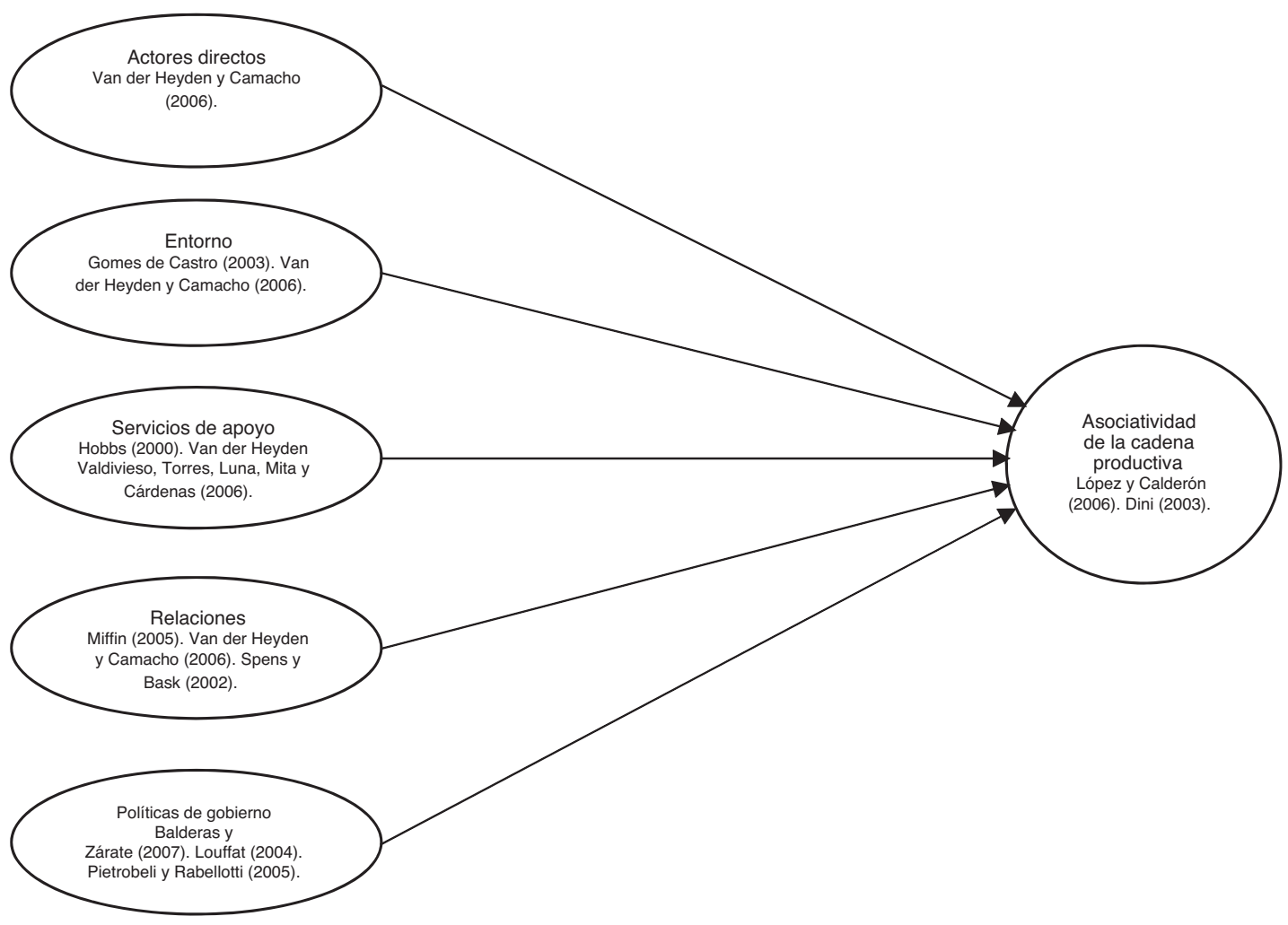

Figura 1. Modelo ex ante.

Fuente: elaboración propia con base en el marco teórico de esta investigación. 

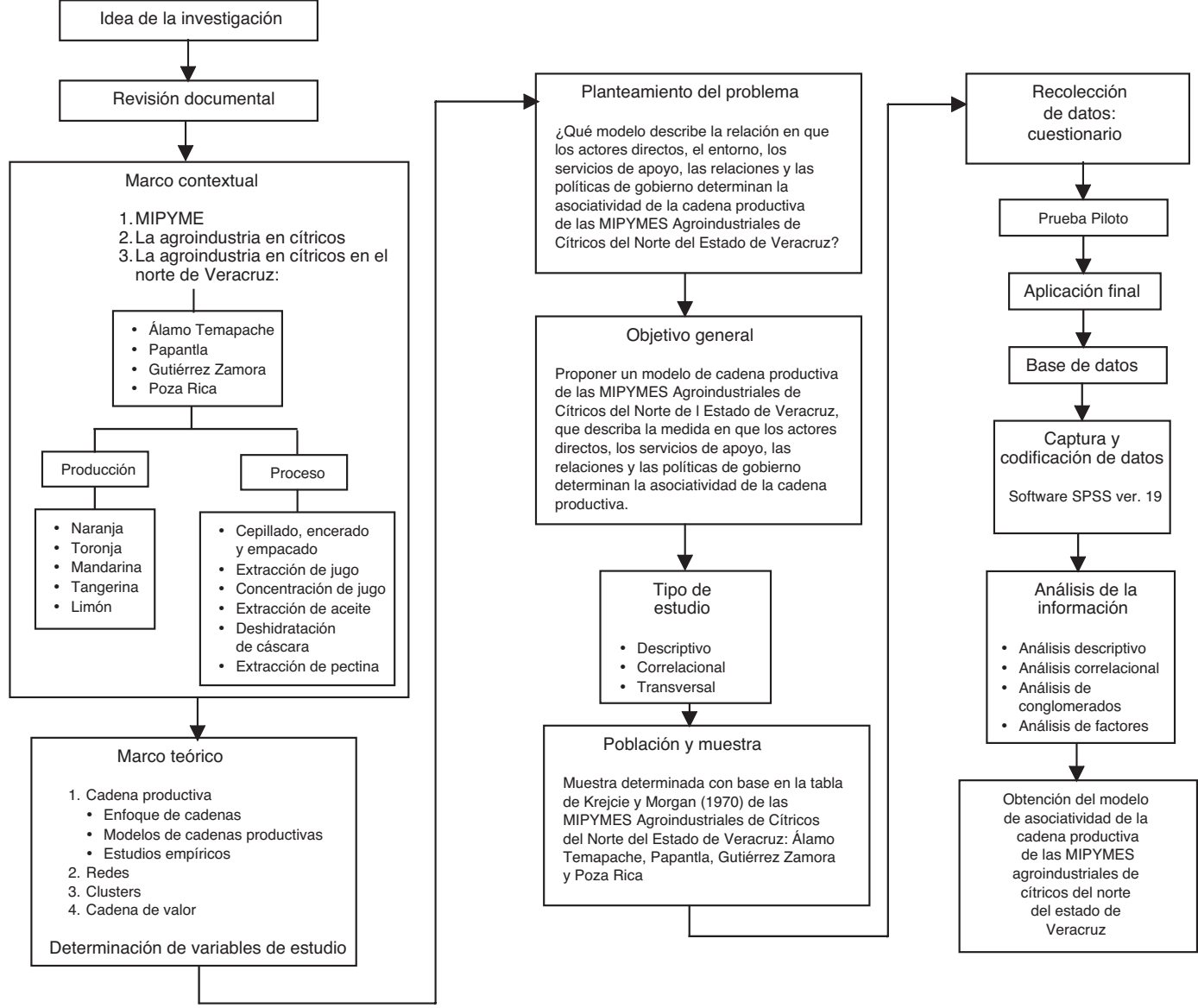


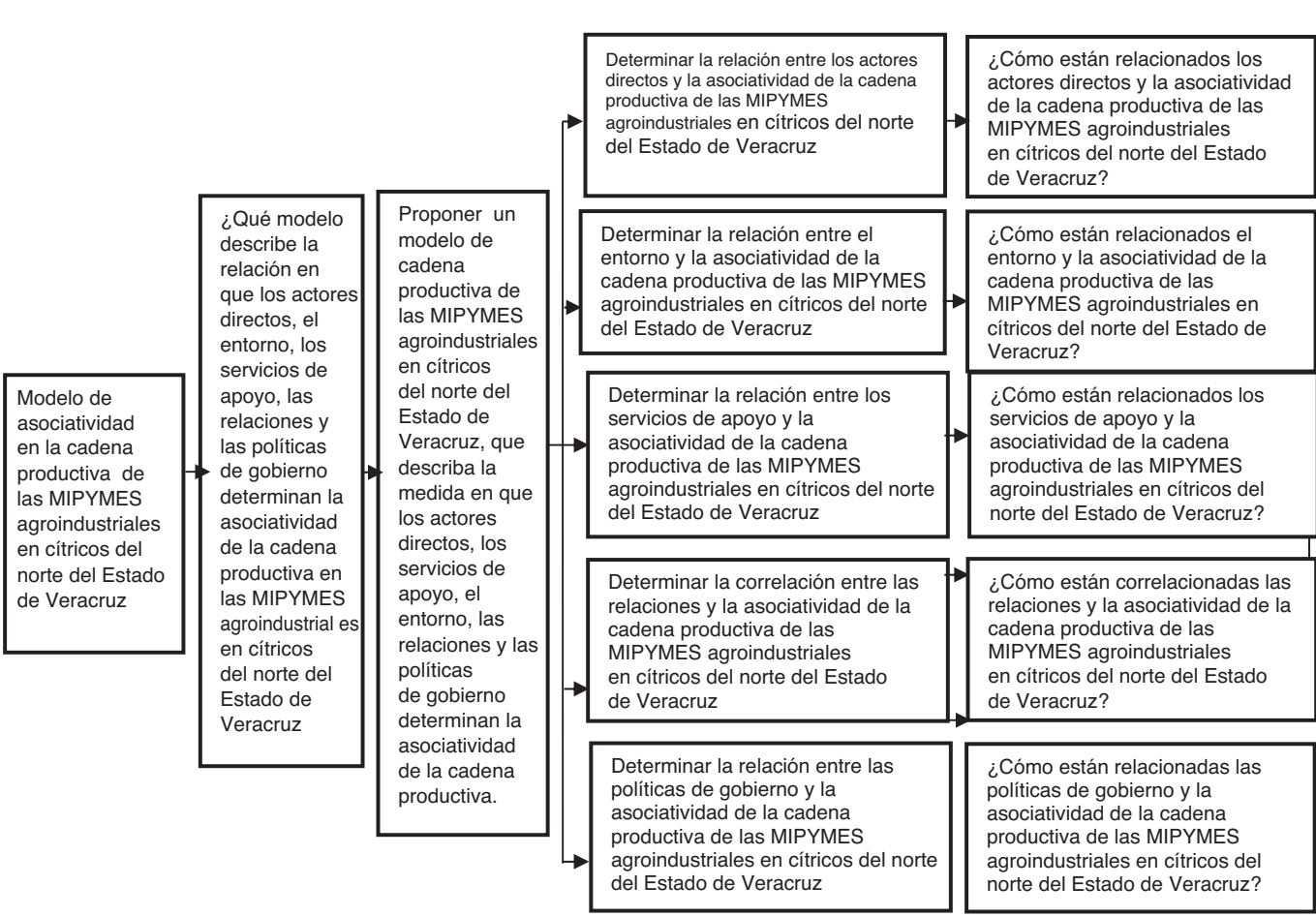

En el modelo de cadena productiva de las MIPYMES agroindustriales en cítricos del norte del Estado de Veracruz, su asociatividad ascrita por los por los actores directos, el entorno, los servicios de apoyo, las relaciones y las políticas de gobierno.

Figura 3. Matriz de congruencia metodológica.

Fuente: elaboración propia con base en la metodología de la investigación. 
Tabla 2

Coeficiente de determinación $\mathrm{R}^{2}$

\begin{tabular}{lllll}
\hline Modelo & $\mathrm{R}$ & $\mathrm{R}^{2}$ & Ajuste de $\mathrm{R}^{2}$ & Error estándar en la estimación \\
\hline 1 & $0.997(\mathrm{a})$ & 0.993 & 0.992 & 2.015
\end{tabular}

Fuente: elaboración propia con base en la resolución del coeficiente de determinación $\mathrm{R}^{2}$.

Tabla 3

Matriz de coeficiente de correlación de Pearson (r)

\begin{tabular}{lllllll}
\hline & Actores directos & Entorno & Servicios de apoyo & Relaciones & Políticas de gobierno & Relaciones \\
\hline Actores directos & 1 & 0.050 & 0.679 & 0.217 & 0.450 & 0.892 \\
Entorno & 0.050 & 1 & 0.189 & 0.263 & 0.127 & 0.522 \\
Servicios de apoyo & 0.679 & 0.189 & 1 & 0.139 & 0.477 & 0.837 \\
Relaciones & 0.217 & 0.263 & 0.139 & 1 & 0.178 & 0.424 \\
Políticas de gobierno & 0.450 & 0.127 & 0.477 & 0.178 & 1 & 0.607 \\
Asociatividad & 0.892 & 0.522 & 0.837 & 0.424 & 0.607 & 1 \\
\hline
\end{tabular}

Fuente: información obtenida de la investigación de campo.

ción. La escala utilizada fue de razón tipo Likert. En el cuestionario se manejaron afirmaciones y juicios con dirección positiva y negativa, con 5 alternativas de respuesta ${ }^{3}$.

Se aplicó la prueba piloto a 10 empresas y el cuestionario final a 38 empresas. En lo que se refiere a la confiabilidad del instrumento de medición, se realizó a la prueba piloto y se obtuvo un alfa de Cronbach $=0.869$, que quiere decir que nuestro instrumento de medición es confiable.

Fueron utilizados el paquete estadístico SPSS versión 19 para las pruebas estadísticas y posteriormente el programa LISREL 8.8 utilizando la modelación mediante ecuaciones estructurales para ajustar el modelo final.

\section{Análisis de resultados}

Para la comprobación de hipótesis se utilizó el coeficiente de determinación $\mathrm{R}^{2}$, como muestra la tabla 2. La variable asociatividad de la cadena productiva obtuvo un valor de 0.993 , por lo que tiene un grado de significancia alto; con base en el resultado obtenido, la asociatividad de la cadena productiva es explicada por los actores directos, entorno, servicios de apoyo y políticas de gobierno.

Los resultados de las correlaciones de Pearson y el coeficiente de determinación $\mathrm{R}^{2}$ presentaron correlaciones significativas (tablas 3 y 4); los valores de las variables independientes oscilan entre 0.424 y 0.892 , correlación de Pearson, y entre 0.402 y 0.796 la correlación $\mathrm{R}^{2}$, lo que significa que tienen una correlación moderada sustancial y marcada alta de cada una de las variables independientes con la variable dependiente asociatividad. Las variables independientes en su conjunto explican el 0.993 , y $\mathrm{R}^{2}$ la asociatividad de la cadena productiva (variable dependiente). La tabla 3 resume la evidencia estadística encontrada.

En cuanto al coeficiente de determinación, tuvo altas correlaciones en los actores directos, los servicios de apoyo y la asociatividad, según se muestra en la tabla 4.

En el análisis de factores (tabla 5) se obtuvieron dos factores; el primer factor está constituido por 3 variables: asociatividad, actores directos y servicios de apoyo, lo que podría representar la

\footnotetext{
${ }^{3}$ La escala de medición tiene los valores de 5 a 1.
} 
Tabla 4

Matriz de coeficiente de determinación $\mathrm{R}^{2}$

\begin{tabular}{ll}
\hline Variables & Coeficiente de determinación $\mathrm{R}^{2}$ \\
\hline Actores directos & 0.796 \\
Entorno & 0.404 \\
Servicios de apoyo & 0.701 \\
Relaciones & 0.402 \\
Políticas de gobierno & 0.468 \\
Asociatividad & 0.992
\end{tabular}

Fuente: información obtenida de la investigación de campo.

Tabla 5

Matriz de componentes (matriz de estructura factorial)

\begin{tabular}{lrr}
\hline & \multicolumn{2}{c}{ Componente } \\
\cline { 2 - 3 } & 1 & 2 \\
\hline Asociatividad & 0.992 & -0.001 \\
Actores & 0.956 & -0.278 \\
Entorno & 0.526 & 0.954 \\
Servicios de apoyo & 0.948 & -0.215 \\
Relaciones & 0.425 & 0.943 \\
Políticas de gobierno & 0.687 & 0.944 \\
\hline
\end{tabular}

Fuente: información obtenida de la investigación de campo.

Tabla 6

Resultados del análisis de ecuaciones estructurales

\begin{tabular}{lc}
\hline Pruebas & Resultados \\
\hline Ji cuadrada & 35.1 \\
Grados de libertad & 59 \\
Nivel de significación & 0.99 \\
RMSEA & 0.00 \\
GFI & 0.870 \\
NFI & 0.762 \\
NNFI & 1.258 \\
CFI & 1.00 \\
\hline
\end{tabular}

Fuente: información obtenida de la investigación de campo.

interacción significativa de estas variables en la cadena productiva. El segundo factor recoge el grupo de variables: entorno, relaciones y políticas de gobierno, por lo que podría representar los elementos que están de soporte en la cadena productiva; ambos factores tienen relación con la cadena productiva.

Una vez aplicado el análisis factorial se encontraron las siguientes cargas factoriales aplicando el método de extracción: Análisis de componentes principales.

Finalmente, y al aplicar el moldeamiento de ecuaciones estructurales, se emplearon las pruebas ji cuadrada, aproximación de la raíz cuadrada media del error (RMSEA), índice de bondad de ajuste (GFI) e índice de ajuste comparativo (CFI). Jaccard y Wan (1996) recomiendan que se realicen tres pruebas de las antes mencionadas; por otro lado, Kline (1998) propone que como mínimo se consulten cuatro. En la tabla 6 se muestra el resultado de estas pruebas; en lo que 


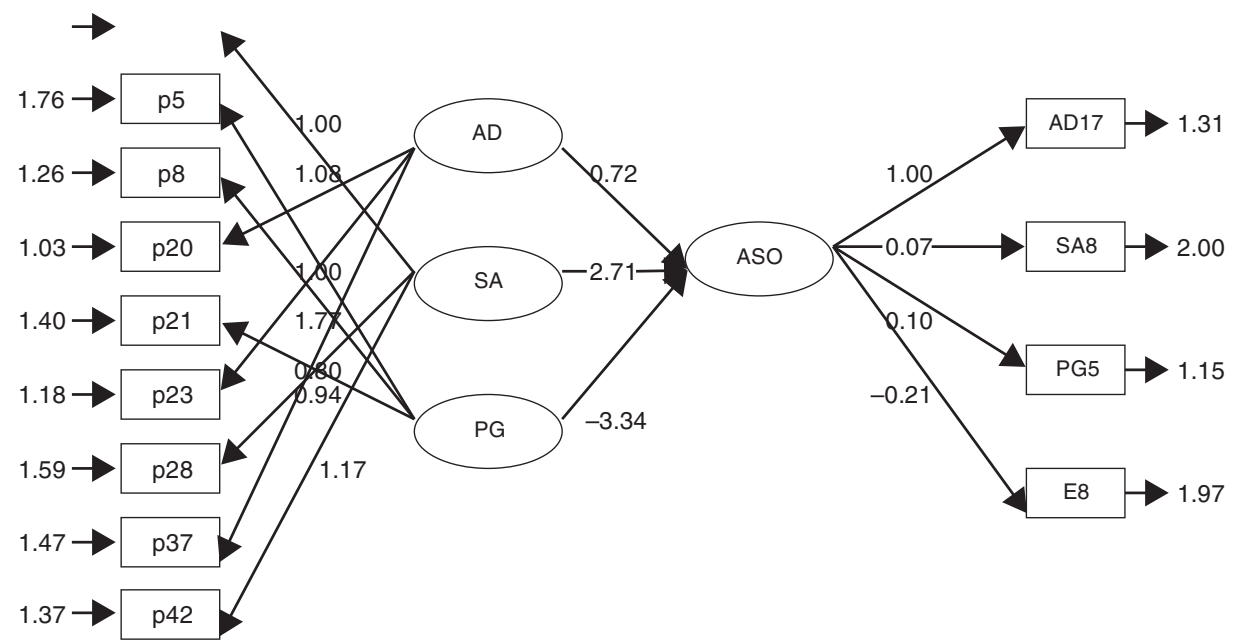

Figura 4. Modelamiento de ecuaciones estructurales.

Los óvalos son las variables latentes o constructos, donde AD es Actores Directos, SA es Servicios de Apoyo, PG es Políticas de Gobierno y ASO es Asociatividad de la Cadena Productiva. Los rectángulos representan los ítems o variables observadas; las flechas que unen los óvalos y los rectángulos son cargas factoriales, las flechas que unen los óvalos son coeficientes beta y las flechas pequeñas que están al lado de los rectángulos son errores de estimación.

Fuente: elaboración propia con base en la investigación de campo.

respecta a ji cuadrada es significativa, porque es mayor de 0.05; el dato de RMSEA es menor de 0.08 , por lo que es satisfactorio; el GFI indica un valor de 0.870 , el cual apoya al modelo porque su valor se acerca a 0.90 ; el CFI cercano a 1 indica un buen ajuste, por lo que los valores superiores a 0.90 se consideran aceptables. Los resultados reportados en la tabla 6 apoyan el ajuste entre el modelo teórico y los datos empíricos.

En la figura 4 se aprecia el modelamiento de ecuaciones estructurales donde la variable dependiente Asociatividad de la cadena productiva es explicada por los Actores directos, Servicios de apoyo y Políticas de gobierno, quienes obtuvieron un alfa de $0.764,0.834$ y 0.608 , respectivamente, como se muestra en la figura 4 , a través de óvalos ${ }^{4}$. Las variables Relaciones y Entorno obtuvieron un alfa de 0.36 y 0.041 , que no contribuyen a la predicción de la variable independiente, esto es por su baja confiabilidad alfa y el tamaño de la muestra.

Con base en lo anterior, de acuerdo a la variable Entorno, Gomes de Castro (2003) y Van der Heyden y Camacho (2006) establecen que se debe incluir el entorno en una cadena productiva debido a que los procesos climáticos, culturales y económicos inciden en el desarrollo de la cadena productiva, y de acuerdo al contexto que se está manejando se considera el proceso productivo desde los insumos, procesamiento, producto final de los cítricos hasta la comercialización nacional e internacional de los mismos. En este sentido, el entorno en la cadena productiva es muy importante; podemos decir que este aspecto explica su baja confiabilidad, porque en realidad los mercados de compradores están fuera del contexto así como la mayoría de los proveedores, que suelen estar centralizados tanto por el gobierno estatal como por el federal.

En lo que respecta a la variable Relaciones, Miffin (2005), Louffat (2004) y Pietrobelli y Rabellotti (2005) consideran que las relaciones son las conexiones de compraventa, sociales y de

\footnotetext{
${ }^{4}$ En el programa LISREL, cuando una variable se subdivide en dimensiones procede a calcular un coeficiente global de alfa de Cronbach por cada variable, como es el caso en lugar de calcular uno para cada constructo.
} 


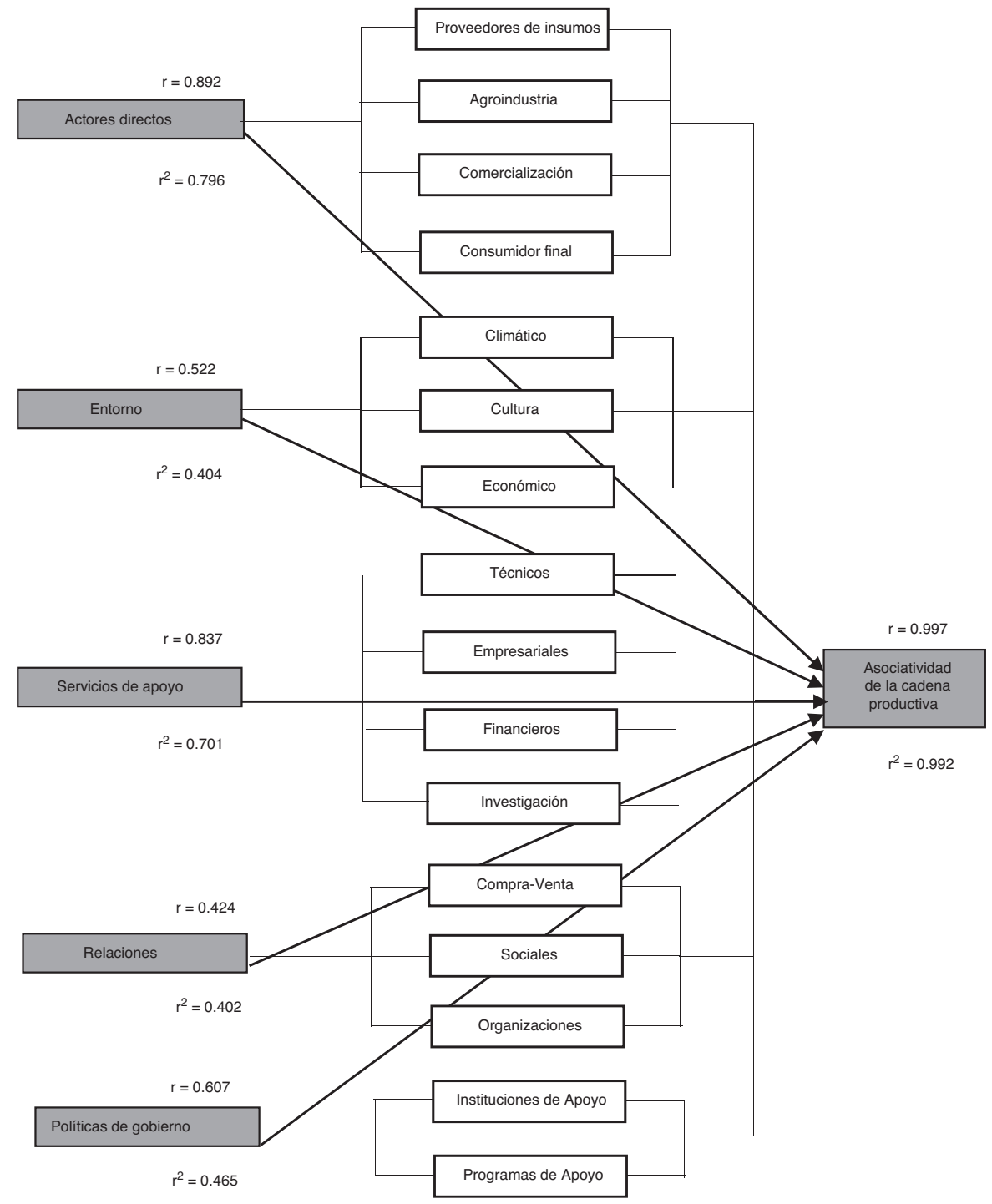

Figura 5. Diagrama ex post facto.

Fuente: elaboración propia con base en el marco teórico y pruebas estadísticas de correlación de Pearson y $\mathrm{R}^{2}$ de esta investigación.

organización que existen en los elementos que forman y participan en la cadena productiva, donde forman las redes en participación en cada estabón en la cadena, por lo que su baja confiabilidad se explica porque aunque se ha pretendido implantar clusters, la desconfianza tradicional y la desconfianza de los productores a los otros actores productivos no generan las sinergias necesarias.

En este sentido, con base en el sustento teórico de las variables Entorno y Relaciones, son consideradas para este modelo. 
Tomando como base la información cuantitativa y cualitativa, se establece el modelo ex post facto (fig. 5), donde se muestra que las relaciones entre las variables son significativas, lo cual explica la realidad de la cadena productiva de las MIPYMES agroindustriales en cítricos del norte del estado de Veracruz. En cuanto al grado de significancia que arrojaron las correlaciones de Pearson y $\mathrm{R}^{2}$, las correlaciones van del valor más elevado (0.992, que significa que existe una correlación altísima muy significativa) en la variable dependiente asociatividad, hasta el valor más bajo (0.402, correlación moderada sustancial) en la variable relaciones.

Teniendo las variables que van a estar en el modelo y con base en matrices de información de cada variable ${ }^{5}$, se realiza el mapeo de la cadena productiva priorizando el actor: procesamiento, por la agroindustria en cítricos.

El modelo propuesto en esta investigación es de tipo matemático (fig. 6). El primero porque, con base en las pruebas estadísticas, se determinan las variables del modelo a través de las relaciones que existan entre ellas. El segundo muestra el estado real del objeto a la hora de la inspección.

La utilidad del modelo es exponer el funcionamiento de la asociatividad en la cadena productiva de las MIPYMES y proponer alternativas para generar una mayor cooperación o coalición de las empresas que interactúan para obtener beneficios mutuos.

\section{Conclusiones}

México es el quinto productor del mundo en cítricos, y Veracruz es el estado de la república de mayor importancia en la producción de los mismos. Dentro de Veracruz, los municipios del norte —Álamo Temapache, Papantla, Gutiérrez Zamora y Poza Rica- son los principales productores y donde existe la mayor parte de las plantas procesadoras de cítricos. Pese a ello, no existen estudios empíricos sobre la asociatividad de las MIPYMES en cítricos, fuera de estudios descriptivos de dependencias oficiales.

Aunque los estudios previos señalan asociaciones positivas entre los actores directos, el entorno, los servicios de apoyo, las relaciones entre productores y las políticas de gobierno como determinantes de la asociatividad, en el caso de México las correlaciones de Pearson, el coeficiente de determinación, el análisis factorial exploratorio y la modelación mediante ecuaciones estructurales encontraron de manera sorpresiva que en caso de las MIPYMES estudiadas solo el trabajo de los propios actores, los servicios de apoyo y las políticas de gobierno tienen influencia sobre la asociatividad. El entorno y las relaciones señalaron bajas asociaciones.

El modelo de asociatividad propuesto en la cadena productiva es un sistema productivo donde los actores directos que lo forman son los productores de cítricos, la agroindustria, la comercialización y los consumidores finales; el actor que tiene relevancia en el modelo es la agroindustria, y dentro de este eslabón se encuentran las MIPYMES agroindustriales en cítricos, que son las cepilladoras, las empacadoras y las enceradoras (comúnmente llamadas por los originarios de la región como empacadoras), empresas procesadoras de jugo simple, plantas jugueras y plantas deshidratadoras y procesadoras de pectina. Las empacadoras reciben los insumos de los productores de cítricos de la región y realizan procesos agroindustriales simples, esto quiere decir que solo cepillan, lavan, enceran y empacan los cítricos ya sea a granel (sin encerar) o en cajas de madera (enceradas); el $98 \%$ son microempresas y el 2\% son pequeñas empresas. De estos cítricos, el 50\% se destina al mercado nacional, principalmente a la central de abastos de la ciudad de México D.F y Guadalajara, y a las tiendas de autoservicio como Walmart, Chedraui, Gigante y Sams. El

\footnotetext{
${ }^{5}$ Estas matrices recogen información cualitativa de cada variable.
} 


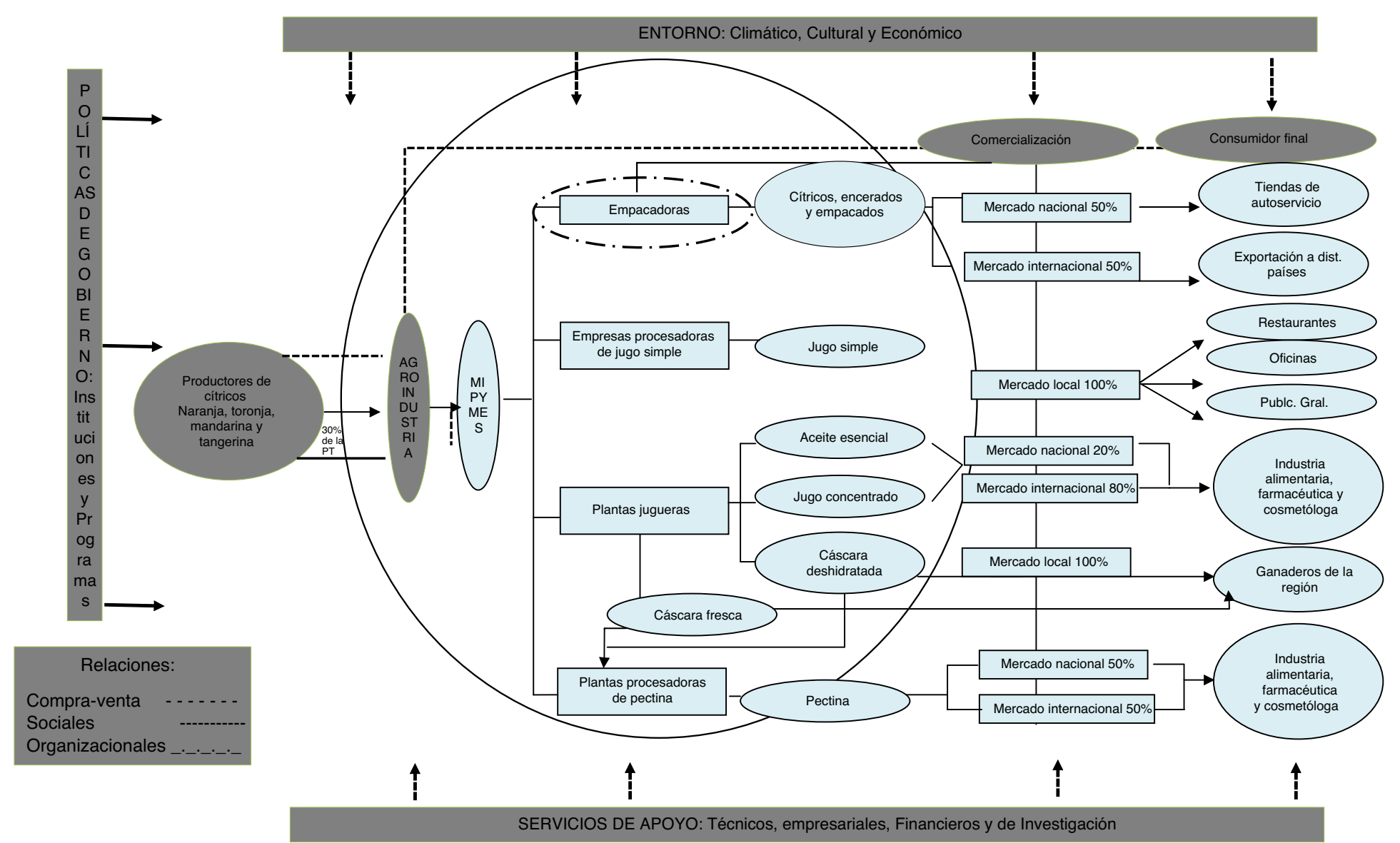

Figura 6. Modelo de asociatividad en la cadena productiva de las MIPYMES agroindustriales en cítricos del norte del estado de Veracruz.

Fuente: elaboración propia con base en los resultados del cuestionario y las matrices de información de las variables. 
$50 \%$ restante es fruta de exportación, que se destina principalmente a Estados Unidos Canadá y Francia.

En cuanto a las empresas procesadoras de jugo, sus insumos los obtienen de los productores de cítricos que están principalmente en la misma ciudad y sus productos son jugos frescos de mandarina, toronja, tangerina y naranja; el $100 \%$ de estas empresas son microempresas formadas de 2 a 6 empleados. Con respecto a la comercialización de sus productos, el 100\% se destina al mercado local, como restaurantes, oficinas y público en general.

En lo que se refiere a las plantas procesadoras de jugo de cítricos (comúnmente llamadas «jugueras» por los habitantes de la región), los insumos para su procesamiento los compra a los productores de cítricos de la región, pero como requieren grandes cantidades de toneladas diarias para su procesamiento, tienen que estar contactando la fruta con anticipación; sus productos son jugo concentrado congelado, aceite esencial y cáscara deshidratada de naranja, toronja, mandarina, tangerina y limón; el $100 \%$ de estas son medianas empresas. El nivel de procesamiento o molienda oscila entre las 500 y 250 toneladas diarias en temporada alta y depende de la capacidad de producción de cada planta. No todas las plantas procesadoras realizan los tres productos mencionados: unas solo se dedican a realizar jugo concentrado de cítricos. En lo que se refiere al jugo concentrado y al aceite esencial, su comercialización se destina el $20 \%$ al mercado nacional y el $80 \%$ al mercado internacional, principalmente a Estados Unidos, Holanda, Alemania e Israel, a las industrias alimentaria, farmacéutica y cosmetóloga principalmente. En lo que respecta a la cáscara deshidratada, solo una de estas empresas la deshidrata y la vende a los ganaderos de la región como alimento balanceado para ganado y su mercado es $100 \%$ local; también vende este producto a las empresas deshidratadoras de cáscara para realizar pectina o la tira en solares baldíos, ríos y arroyos, ocasionando contaminación al medio ambiente.

En lo que respecta a las plantas deshidratadoras de cáscara, el 100\% (2 empresas) son pequeñas empresas; sus insumos para procesar (cáscara o gabazo en fresco) los obtienen en las plantas jugueras, y estas empresas los deshidratan y los convierten en pectina. La comercialización de sus productos se realiza el $50 \%$ en el mercado nacional y el $50 \%$ en el mercado internacional, a las industrias alimentaria, farmacéutica y cosmetóloga.

\section{Referencias}

Carranza, R., Macedo, R., Cámara, J., Sosa, J., Meraz, A. y Valdivia, A. (2007). Competitividad en la cadena productiva de leche del Estado de Aguascalientes, México. Revista Agrociencia, 41(6), 701-709. Disponible en: www.redalyc.org/articulo.oa?id=30241611

Comisión Económica Para América Latina y el Caribe. (2003). Apertura económica y encadenamientos productivos [consultado 27 Mar 2007]. Disponible en: www.enclac.org ISBN: 9213218273

Croxton, K., García-Dastugue, S., Lambert, D. y Rogers, D. (2001). The supply chain management processes. International Journal of Logistics Management, 12(2), 13-33, http://doi.org/10.1108/09574090110806271

Cuevas, V., Espinosa, J., Flores, A., Romero, F., Vélez, A., Jolalpa, J. y Vázquez, R. (2007). Diagnóstico de la cadena productiva de leche de vaca en el Estado de Hidalgo, México. Revista Inifab, 45(1), 25-40. Disponible en: http://www.redalyc.org/articulo.oa?id=61345103 ISSN 0040-1889.

Diario Oficial de la Federación. (2009). Ley para el desarrollo de la competitividad de la micro, pequeña y mediana empresa. México Decreto [consultado 8 Sep 2012]. Disponible en: www.dof.gob.mx

Dini, M. (2003). Plan operativo para el fomento de los clusters [consultado 12 Jul 2009]. Disponible en: http://cepal.org/publicaciones/xml/9/32009/S180DP-L2788-P

Gereffi, G. (1999). International trade and industrial upgrading in the apparel commodity chain. Journal of International Economics, 48(1), 37-70, http://doi. org/10.1016/S0022-1996 (98) 00075-0.

Gomes de Castro, A. (2003). Foresight study on the productive chain on the fishery industry in the region den South American Pacific Coast [consultado 12 Ago 2008]. Disponible en: http://unido.org/fileadmin/import23306 
Guaipatín, C. (2004). Orientación para la evaluación de proyectos de integración productiva [consultado 9 Sep 2008]. Disponible en: http://www.iadb.org/wmsfiles/products/publications/documents/368413.pdf

Instituto Tecnológico de Estudios Superiores de Monterrey. (2004). Estudio sobre cadena productiva para la seguridad alimentaria y desarrollo rural [manuscrito no publicado].

Jaccard, J. y Wan, C. (1996). LISREL. Análisis de los efectos de interacción en la regresión múltiple. Newbury Park, Londres: Sabio.

Kaplinsky, R. (2000). Spreading the gains from globalization: What can be learned value chain analysis. Journal: Problems of Economic Transition, 47(2), 74-115, http://doi. org/10.1080/10611991.2004.11049908. Disponible en: http://mesharpe.metapress.com

Kline, R. (1998). Principles and Practice of Structural Equation Modeling. New York, NY: The Guilford Press.

Krejcie, R. y Morgan, D. (1970). Statistics for Business. Mac Graw Hill: Estados Unidos.

Lazzarini, S., Chaddad, F. y Cook, M. (2001). Integrating supply chain and network analyses: The study of net-chains. Wegeningen Academic publishers., 1(1), 7-22 [consultado 23 Nov 2014]. http://doi. org//10.3920/JCNS2001.x002. Disponible en: http://wageningenacademic.metapress.com/content/33550414t42t0q06/\#.VMbACGSG90Q

López, L. y Calderón, G. (2006). Análisis de las dinámicas culturales al interior de un clúster empresarial. Revista Estudios Gerenciales, 99, 13-37 [consultado 15 Ene 2015]. Disponible en: http://www.redalyc.org/articulo.oa?id=21209901

Louffat, E. (2004). Interconexión entre redes organizacionales, alianzas estratégicas y negociaciones: un estudio multicaso. Journal of Economics Finance and Administrative Science, 9(16), 103-126 [consultado 20 Dic 2014]. Disponible en: http://jefas.esan.edu.pe/indez.php/jefas/article/view/158. ISSN: 2218-0648 (on line). ISSN: 2077-1886 (printed).

Miffin, I. (2005). Redes empresariales y clusters: teoría y ejemplos en el caso peruano. [consultado 12 Ago 2009]. Disponible en: http://www.prompyme.gob.pe/upload/publicación_20-9-2006-3.pdf

Ochoa, D. y Montoya, L. (2010). Consorcios microbianos: una metáfora biológica aplicada a la asociatividad empresarial en cadenas productivas agropecuarias. Revista Facultad de Ciencias Económicas: Investigación y Reflexión, 18(2), 55-74. Print version ISSN 0121-6805.

Pietrobelli, C. y Rabellotti, R. (2005). Mejora de la competitividad en clusters y cadenas productivas en América Latina, el papel de las políticas [consultado 18 Jul 2009]. Disponible en: http://www.iabd.org/sds/msm

Programa Estatal de Ciencia y Tecnología del Estado de Jalisco. (2007). Programa Estatal de Ciencia y Tecnología del Estado de Jalisco, México, 2007 [consultado 08 Ago 2008]. Disponible en: www.jalisco.gob.mx

Romero, D. (2001). La agroindustria en Veracruz ante la globalización. México: Arana Editores.

Secretaria de Agricultura, Ganadería, Desarrollo Rural, Pesca y Alimentación. (2014). México entre los líderes en producción de cítricos a nivel mundial [consultado 26 Nov 2014]. Disponible en: http://www.sagarpa.gob.mx/Delegaciones/sanluispotosi/boletines/Paginas/BOL1301112.aspx

Sistema de Información Agropecuaria y Pesquera (2014). Anuario estadístico de producción agrícola. [consultado 5 Nov 2015]. Disponible en: http://www.siap.gob.mx/cierre-de-la-produccion-agricola-por-estado/

Sistema de Información Empresarial. (2014). Estadísticas por sector y tamaño de empresa [consultado 2 Nov 2014]. Disponible en: http://www.siem.gob.mx/siem/portal/consultas/respuesta.asp?estado=30

Van der Heyden, D. y Camacho, P. (2006). Guía metodológica para el análisis de cadenas productivas 2006 [consultado 8 Sep 2009]. Disponible en: http//:www.ruralfinance.org/fileadmin/templates/rflc/documents/51825

Wisner, J. (2003). A structural equation model of supply chain management strategies and firm performance. Journal of Business Logistics, 24(1), 1-26 [consultado 15 Ene 2013]. http://doi.org/10.1002/j.2158-1592.2003.tb00030.x. Disponible en: http://onlinelibrary.wiley.com/doi/10.1002/j.21581592.2003.tb00030.x/abstract 